\title{
Stereoselective Total Synthesis of the Putative Structure of Nitraraine
}

Federica Arioli, Maria Pérez, Fabiana Subrizi, Núria Llor, Joan Bosch and Mercedes Amat ${ }^{*}$

Laboratory of Organic Chemistry, Faculty of Pharmacy, and Institute of Biomedicine (IBUB), University of Barcelona, 08028-Barcelona, Spain.

amat@ub.edu

\section{Abstract}

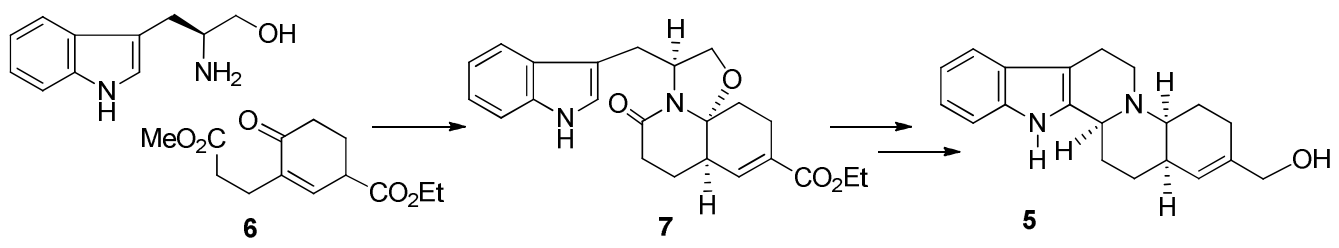

After the structure originally proposed for nitraraine was shown to be incorrect by total synthesis, the alternative structure $\mathbf{5}$ was recently suggested for the alkaloid on biosynthetic grounds and by comparison with the ${ }^{1} \mathrm{H}$ NMR data of tangutorine. The unambiguous synthesis of $\mathbf{5}$ is reported from tryptophanol and ketodiester $\mathbf{6}$, via oxazoloquinolone lactam 7. However, the melting point and ${ }^{1} \mathrm{H}$ NMR data of $\mathbf{5}$ did not match those reported for the natural product.

Nitraraine $^{1}$ is an indole alkaloid isolated in 1985 from Nitraria schoberi L., collected in Kyzyl-Kum (Uzbekistan). On the basis of its mass-spectrometric fragmentation, spectroscopic UV, IR and ${ }^{1} \mathrm{H}$ NMR data, chemical transformations and correlations, and degradation studies, the yohimbane-type structure $\mathbf{1}$ was assigned to nitraraine (Figure 1). Catalytic hydrogenation of nitraraine afforded dihydronitraraine (assigned as 2), which had also been isolated from the same plant. ${ }^{2}$ Some years later, two new alkaloids, $O$-acetylnitraraine ${ }^{3}$ and nitraraidine, ${ }^{4}$ were also isolated from Nitraria species, and their structures were assigned as $\mathbf{3}$ and $\mathbf{4}$, respectively, mainly by chemical correlations with nitraraine and dihydronitraraine. 

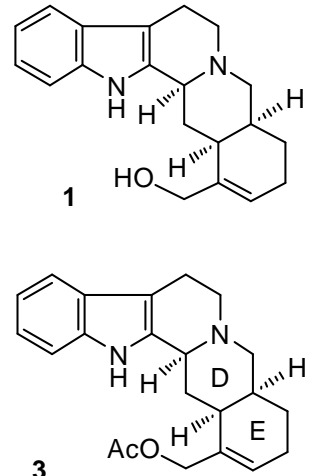
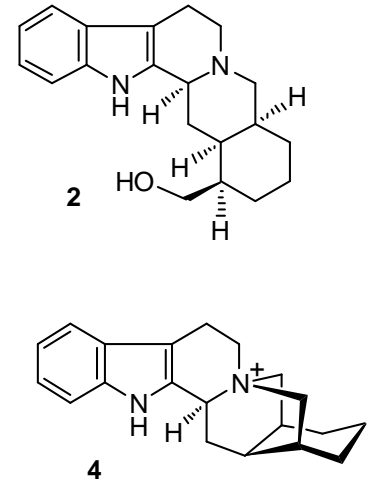

Figure 1. Structures proposed for nitraraine, dihydronitraraine, $O$-acetylnitraraine, and nitraraidine.

A pentacyclic alcohol with the structure 1 had previously been prepared ${ }^{5}$ by $\mathrm{LiAlH}_{4}$ reduction of apo$\alpha$-yohimbine. However, the data reported for this alcohol 1 (obtained as the hydrochloride) did not allow the proposed structure for nitraraine (isolated as the base) to be corroborated.

Later on, three different syntheses of pentacyclic alcohol $\mathbf{1}$, either in enantiopure form ${ }^{6,7}$ or as a racemate, ${ }^{8}$ were reported. The melting point and ${ }^{1} \mathrm{H}$ NMR data of $\mathbf{1}$ were significantly different from those reported for nitraraine. Similarly, the melting point of synthetic $\mathbf{2}$ differed $^{6}$ from that of the alkaloid dihydronitraraine. ${ }^{2}$ Consequently, a reasonable doubt arose about the correct structure of the alkaloids of the nitraraine family.

In 2011, Poupon et al. published ${ }^{9}$ an excellent and comprehensive article that provides a detailed analysis of the data available for nitraraine and the above-mentioned nitraraine-related alkaloids, suggesting pentacyclic alcohol $\mathbf{5}$, a structural isomer of $\mathbf{1}$, as a possible structure for nitraraine. The proposal was based on biosynthetic considerations ${ }^{10}$ and a comparison of the physical and spectroscopic data reported for these alkaloids with those of tangutorine ${ }^{11}$ and its $O$-acetyl and dihydro derivatives (Figure 2).
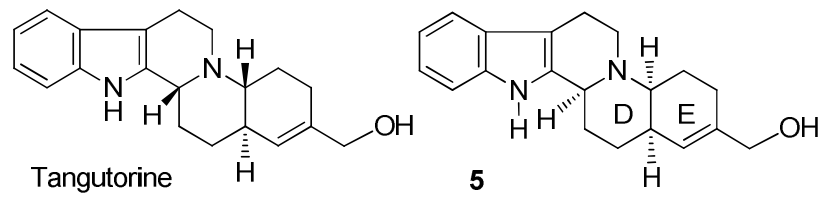

Figure 2. Structure of tangutorine and putative structure of nitraraine. 
Both nitraraine and tangutorine are alkaloids isolated from Nitraria species (Nitraria schoberi and Nitraria tangutorum, respectively), which belong to the Nitrariaceae family, whereas yohimbine-type alkaloids have a monoterpenoid origin and are found in plants of the Rubiaceae, Loganiaceae and Apocinaceae families. Moreover, the specific rotation reported for both alkaloids is zero, which could be attributed to similar biosynthetic pathways that do not involve the monoterpene secologanin. On the other hand, nitraraine, tangutorine, and their $O$-acetyl derivatives each have an olefinic proton that resonates at a very similar chemical shift in their ${ }^{1} \mathrm{H}$ NMR spectra. Further, the melting point of these two alkaloids differs by only a few degrees Celsius. Another point of interest is that treatment of dihydronitraraine with $p$-TsCl provides the alkaloid nitraraidine, which is an $N$-quaternary hexacyclic salt, thus pointing to a cis $\mathrm{D} / \mathrm{E}$ ring junction. Taking into account all the above, Poupon proposed the tangutorine diastereoisomer $\mathbf{5}$ as a plausible structure for nitraraine.

In this communication we report the enantioselective total synthesis of $\mathbf{5}$. In the context of our studies on the use of tryptophanol-derived lactams as enantiomeric scaffolds for the synthesis of indole alkaloids, ${ }^{12}$ we visualized a synthetic route to this alcohol. Key steps to assemble the required pentacyclic skeleton would be a stereoselective cyclocondensation of $(S)$-tryptophanol with an appropriately substituted 6-oxocyclohexenepropionate derivative $\mathbf{6}$ and a Bischler-Napieralski cyclization of the resulting oxazoloquinolone lactam $7^{13}$ (Scheme 1). The synthesis would also require the subsequent removal of the hydroxymethyl substituent coming from tryptophanol and the reduction of the ester function. 


\section{Scheme 1. Synthetic strategy}

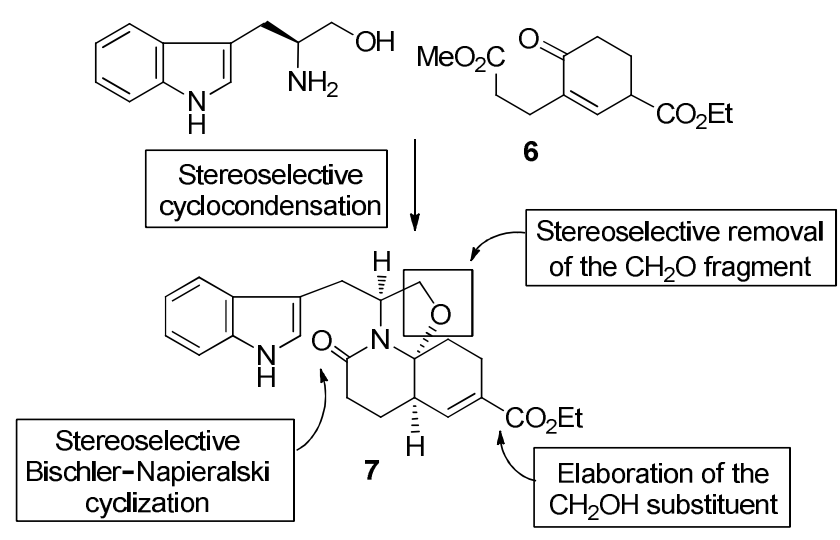

The required $\delta$-keto ester $\mathbf{6}$ was prepared in $56 \%$ overall yield from ethyl 4oxocyclohexanecarboxylate 8, via keto sulfoxide 9, as outlined in Scheme 2.

\section{Scheme 2. Preparation of the starting $\delta$-keto ester 6}

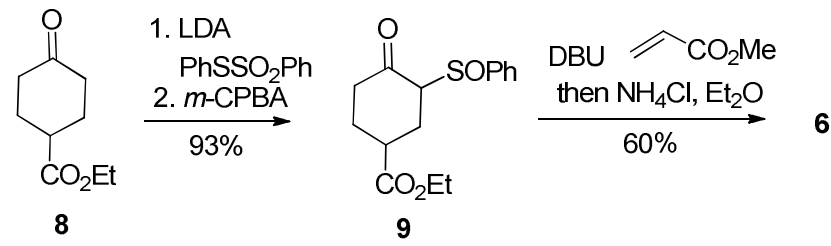

The cyclocondensation reaction of $(S)$-tryptophanol with $\delta$-keto ester $\mathbf{6}$ stereoselectively gave tricyclic lactam 7 in $71 \%$ yield. ${ }^{14}$ Starting from 7 , the closure of the central $\mathrm{C}$ ring was satisfactorily accomplished under classical Bischler-Napieralski reaction conditions. Without purification, treatment of the resulting hexacyclic derivative with $\mathrm{LiAlH}_{4}$ brought about both the reductive opening of the oxazolidine ring to stereoselectively give the required cis-decahydroquinoline ring junction and the reduction of the ester function, leading to the pentacyclic diol derivative $\mathbf{1 0}$ in $60 \%$ overall yield (Scheme 3). In contrast, a similar sequence from $\mathbf{7}^{\mathbf{S}}$ led to the indoloquinolizidine derivative 11, arising from an initial $\alpha$-amidoalkylation reaction on the indole ring ${ }^{15}$ (Scheme 4 ). 


\section{Scheme 3. Synthesis of 5, the Putative Structure of Nitraraine}
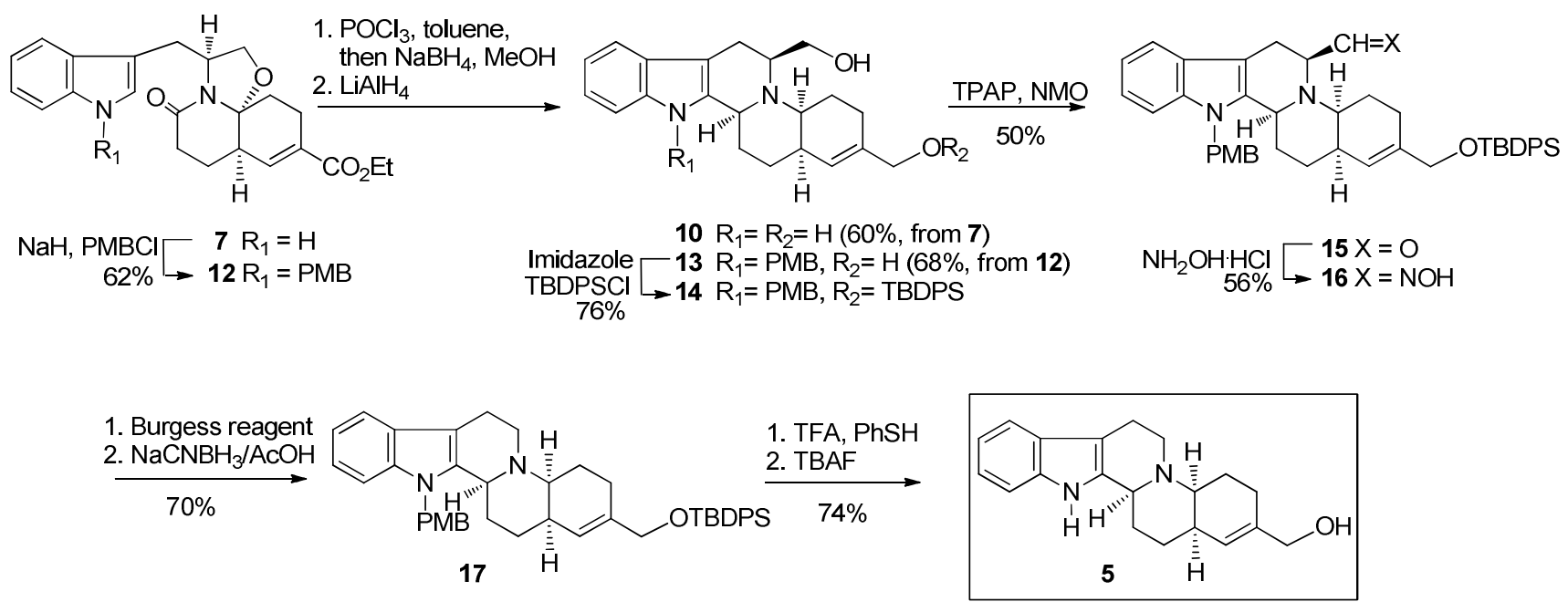

\section{Scheme 4. Cyclization of lactam 7'}

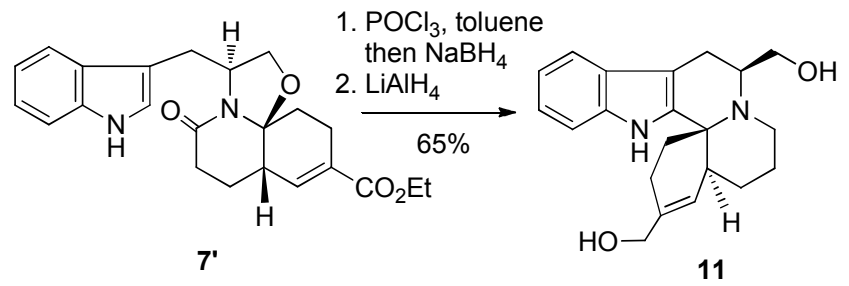

The next stage of the synthesis was the removal of the hydroxymethyl substituent coming from tryptophanol, which required the selective protection of the allylic hydroxy group, but unfortunately the insolubility of diol $\mathbf{1 0}$ precluded its manipulation. For this reason, the indole nitrogen of $\mathbf{7}$ was protected as a $p$-methoxybenzyl derivative, and the resulting lactam 12 was converted to $\mathbf{1 3}$ in $68 \%$ overall yield following the above Bischler-Napieralski cyclization- $\mathrm{LiAlH}_{4}$ reduction sequence.

Once the allylic hydroxyl group was selectively protected with the bulky tert-butyldiphenylsilyl group, the removal of the hydroxymethyl substituent of $\mathbf{1 4}$ was performed in four steps: oxidation to aldehyde 15 using tetrapropylammonium perruthenate in the presence of $N$-methylmorpholine $N$-oxide as the co-oxidant (TPAP/NMO) ${ }^{16}$ subsequent dehydration of the corresponding oxime $\mathbf{1 6}$ with Burgess reagent, and reductive decyanation of the resulting $\alpha$-amino nitrile. 
Finally, deprotection of the indole nitrogen of pentacycle 17 using TFA in the presence of PhSH as a carbocation scavenger, followed by desilylation of the alcohol function gave the target pentacyclic alcohol 5.

Our synthetic product 5 showed mass-spectral peaks with the same $\mathrm{m} / \mathrm{z}$ as natural nitraraine. ${ }^{1}$ However, the melting point $\left(241-242{ }^{\circ} \mathrm{C}\right)$ of 5 and the chemical shift of the olefinic proton in its ${ }^{1} \mathrm{H}$ NMR spectrum $\left(\delta 5.86\right.$ in $\left.\mathrm{CF}_{3} \mathrm{CO}_{2} \mathrm{D}\right)$ were different from those described for the alkaloid $(\mathrm{mp} 280-281$ ${ }^{\circ} \mathrm{C} ; \delta 5.22$ in $\mathrm{CF}_{3} \mathrm{CO}_{2} \mathrm{H}^{17}$ ) (Figure 3).
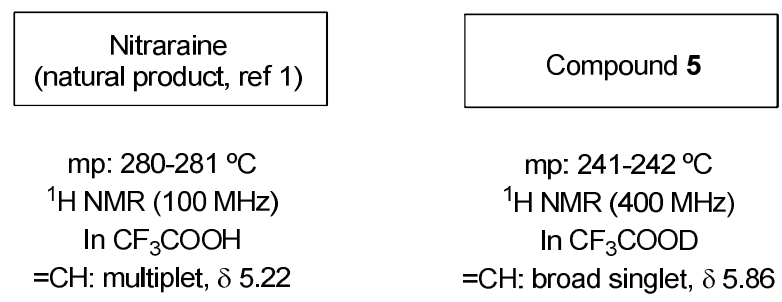

Figure 3. Melting point and ${ }^{1} \mathrm{H}$ NMR data of nitraraine and compound $\mathbf{5}$.

The above data made evident that the real structure of nitraraine and related alkaloids remains an unsolved question and that further synthetic efforts are needed to reach a definitive conclusion.

\section{Experimental Section}

Ethyl 4-Oxo-3-(phenylsulfonyl)cyclohexanecarboxylate (9): First step: LDA (4.41 mL, $8.82 \mathrm{mmol}$ of a $2 \mathrm{M}$ solution in $\mathrm{THF} /$ heptane/ethylbenzene) was added at $-78{ }^{\circ} \mathrm{C}$ to a solution of commercial ethyl 4oxocyclohexanecarboxylate $(\mathbf{8} ; 1.5 \mathrm{~g}, 8.82 \mathrm{mmol})$ in dry THF $(90 \mathrm{~mL})$, and the solution was stirred for 30 minutes. Then $\mathrm{PhSSO}_{2} \mathrm{Ph}(2.21 \mathrm{~g}, 8.82 \mathrm{mmol})$ in THF $(10 \mathrm{~mL})$ was added, and the resulting mixture was stirred at $-78{ }^{\circ} \mathrm{C}$ for 40 minutes. The reaction was quenched with saturated aqueous $\mathrm{NH}_{4} \mathrm{Cl}(50$ $\mathrm{mL}$ ), and the resulting mixture was extracted with EtOAc. The combined organic extracts were dried, filtered, and concentrated to afford ethyl 4-oxo-3-(phenylthio)cyclohexanecarboxylate, which was 
used in the next step without purification: ${ }^{1} \mathrm{H}$ NMR (400 MHz, $\left.\mathrm{CDCl}_{3}\right) \delta 1.27(\mathrm{t}, J=7.2 \mathrm{~Hz}, 3 \mathrm{H}), 1.93$ $2.02(\mathrm{~m}, 1 \mathrm{H}), 2.25-2.29(\mathrm{~m}, 1 \mathrm{H}), 2.31-2.38(\mathrm{~m}, 1 \mathrm{H}), 2.39-2.43(\mathrm{~m}, 2 \mathrm{H}), 2.98-3.03(\mathrm{~m}, 1 \mathrm{H}), 3.04-3.11$ (m, 1H), $3.89(\mathrm{td}, J=4.4,0.8 \mathrm{~Hz}, 1 \mathrm{H}), 4.17(\mathrm{q}, J=7.2 \mathrm{~Hz}, 2 \mathrm{H}), 7.29(\mathrm{~d}, J=7.2 \mathrm{~Hz}, 2 \mathrm{H}), 7.40(\mathrm{dd}, J=$ 8.8, $2.0 \mathrm{~Hz}, 2 \mathrm{H}), 7.56(\mathrm{~d}, J=6.8 \mathrm{~Hz}, 1 \mathrm{H})$. Second step: A solution of $m$-CPBA $(70 \%, 2.17 \mathrm{~g}, 8.82$ $\mathrm{mmol})$ in $\mathrm{CH}_{2} \mathrm{Cl}_{2}(20 \mathrm{~mL})$ was added at $-78^{\circ} \mathrm{C}$ to a solution of the above phenylthio derivative $(2.46 \mathrm{~g}$, $8.82 \mathrm{mmol})$ in $\mathrm{CH}_{2} \mathrm{Cl}_{2}(180 \mathrm{~mL})$. After 5 minutes, the reaction was quenched with the addition of saturated aqueous $\mathrm{Na}_{2} \mathrm{~S}_{2} \mathrm{O}_{3}$. The resulting mixture was extracted with $\mathrm{CH}_{2} \mathrm{Cl}_{2}$, and the combined organic extracts were washed with saturated aqueous $\mathrm{NaHCO}_{3}$ and brine, dried, filtered, and concentrated. Flash chromatography of the residue (4:1 hexane-EtOAc) afforded the sulfonyl derivative 9 (2.38 $\mathrm{g}, 93 \%$ yield for the two steps) as a complex mixture of diastereoisomers. HRMS (ESI-TOF) $m / z$ : $[\mathrm{M}+\mathrm{H}]^{+}$ Calcd for $\mathrm{C}_{15} \mathrm{H}_{19} \mathrm{O}_{4} \mathrm{~S} 295.0999$; Found 295.0999.

Methyl 3-(Ethoxycarbonyl)-6-oxocyclohexenepropionate (6): A solution of DBU (374 mg, 2.45 $\mathrm{mmol}$ ) in DMF (5 mL) was added at $-40^{\circ} \mathrm{C}$, under inert atmosphere, to a solution of the above sulfonyl derivatives 9 (720 mg, $2.45 \mathrm{mmol})$ in DMF (15 mL), and the resulting mixture was stirred for $20 \mathrm{~min}$. A solution of methyl acrylate $(211 \mathrm{mg}, 2.45 \mathrm{mmol})$ in DMF $(5 \mathrm{~mL})$ was then added to the mixture, and the reaction was allowed to reach $10{ }^{\circ} \mathrm{C}$ over $1.5 \mathrm{~h}$. The reaction was quenched with saturated aqueous $\mathrm{NH}_{4} \mathrm{Cl}(20 \mathrm{~mL})$, and the resulting mixture was extracted with $\mathrm{Et}_{2} \mathrm{O}$. The organic layer was dried and filtered, and the filtrate was stirred at room temperature for $20 \mathrm{~h}$. After this time, the solvent was evaporated under reduced pressure. Flash chromatography of the residue (hexane to 9.5:0.5 hexane-EtOAc) afforded oxoester 6 (375 mg, 60\%): IR (film) $v\left(\mathrm{~cm}^{-1}\right) 1678(\mathrm{CO}), 1736$ (CO); ${ }^{1} \mathrm{H}$ NMR (400 MHz, $\mathrm{CDCl}_{3}, \mathrm{COSY}, g$-HSQC) $\delta 1.30\left(\mathrm{t}, J=7.1 \mathrm{~Hz}, 3 \mathrm{H}, \mathrm{CH}_{3}\right.$ ), 2.12-2.23 (m, 1H, H-4'), 2.302.41 (m, 2H, H-2, H-4'), 2.44-2.49 (m, 2H, H-3), 2.53-2.61 (m, 3H, H-2, H-5'), 3.36-3.42 (m, 1H, H3'), 3.66 (s, 3H, $\left.\mathrm{OCH}_{3}\right), 4.21$ (q, $\left.J=7.1 \mathrm{~Hz}, 2 \mathrm{H}, \mathrm{CH}_{2} \mathrm{CH}_{3}\right), 6.85\left(\mathrm{~d}, J=4.4 \mathrm{~Hz}, 1 \mathrm{H}, \mathrm{H}-2{ }^{\prime}\right)$; ${ }^{13} \mathrm{C}$ NMR (100.6 MHz, $\left.\mathrm{CDCl}_{3}\right) \delta 14.1\left(\mathrm{CH}_{3}\right), 25.4(\mathrm{C}-3), 25.7\left(\mathrm{C}^{\prime} 4^{\prime}\right), 32.8$ (C-5'), $36.6(\mathrm{C}-2), 42.0\left(\mathrm{C}-3^{\prime}\right), 51.5$ $\left(\mathrm{OCH}_{3}\right), 61.3\left(\mathrm{CH}_{2} \mathrm{CH}_{3}\right), 132.6\left(\mathrm{C}-1^{\prime}\right), 142.3\left(\mathrm{C}-2^{\prime}\right), 171.8$ (COO), 173.3 (COO), $197.8(\mathrm{CO})$; HRMS (ESI-TOF) $m / z:[\mathrm{M}+\mathrm{H}]^{+}$Calcd for $\mathrm{C}_{13} \mathrm{H}_{19} \mathrm{O}_{5}$ 255.1227; Found 255.1228. 
Ethyl

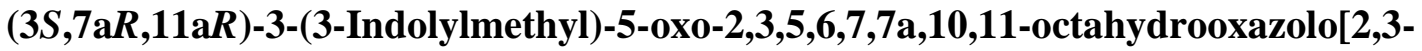

$j$ ]quinoline-9-carboxylate (7): Isobutyric acid $(105 \mu \mathrm{L}, 1.13 \mathrm{mmol})$ was added to a solution of tryptophanol $(97 \mathrm{mg}, 0.51 \mathrm{mmol})$ and oxoester $6(100 \mathrm{mg}, 0.39 \mathrm{mmol})$ in toluene $(8 \mathrm{~mL})$. The mixture was stirred at reflux for $24 \mathrm{~h}$, with azeotropic elimination of water by a Dean-Stark system. The resulting mixture was cooled and concentrated under reduced pressure. The residue was dissolved in EtOAc and washed with saturated aqueous $\mathrm{NaHCO}_{3}$. The combined organic extracts were dried, filtered, and concentrated to give a foam. Crystallization from EtOAc gave lactam 7 (109 mg, 71\%). Evaporation of the solvent afforded the $(3 S, 7 \mathrm{a} S, 11 \mathrm{a} S)$ diastereoisomer (7'; $19 \mathrm{mg}, 17 \%)$. Lactam 7: $[\alpha]^{22}{ }_{\mathrm{D}}+20.2\left(c\right.$ 1.0, $\left.\mathrm{CHCl}_{3}\right) ; \mathrm{IR}($ film $) \vee\left(\mathrm{cm}^{-1}\right) 1629(\mathrm{NCO}), 1714(\mathrm{COO}), 3303(\mathrm{NH}) ;{ }^{1} \mathrm{H}$ NMR $(400$ $\mathrm{MHz}, \mathrm{CDCl}_{3}, \mathrm{COSY}, g$-HSQC) $\delta 1.28\left(\mathrm{t}, J=7.5 \mathrm{~Hz}, 3 \mathrm{H}, \mathrm{CH}_{3}\right), 1.56-1.68(\mathrm{~m}, 2 \mathrm{H}, \mathrm{H}-7, \mathrm{H}-11), 1.95$ $(\mathrm{dm}, J=14.0 \mathrm{~Hz}, 1 \mathrm{H}, \mathrm{H}-7), 2.05-2.10(\mathrm{~m}, 1 \mathrm{H}, \mathrm{H}-11), 2.25-2.30$ (m, 1H, H-7a), 2.33-2.39 (m, 1H, H10), 2.43-2.52 (m, 2H, H-6, H-10), 2.63 (dd, $J=18.4,6.4$ Hz, 1H, H-6), 2.89 (dd, $J=14.0,10.4$ Hz, 1H, $\mathrm{CH}_{2}$-ind), 3.62 (ddd, $J=14.0,3.4,0.8 \mathrm{~Hz}, 1 \mathrm{H}, \mathrm{CH}_{2}$-ind), 3.81 (dd, $J=8.8,8.0 \mathrm{~Hz}, 1 \mathrm{H}, \mathrm{H}-2$ ), 4.01 $(\mathrm{dd}, J=8.8,8.0 \mathrm{~Hz}, 1 \mathrm{H}, \mathrm{H}-2), 4.19\left(\mathrm{q}, J=7.5 \mathrm{~Hz}, 2 \mathrm{H}, \mathrm{CH}_{2} \mathrm{CH}_{3}\right), 4.67(\mathrm{dtd}, J=10.4,8.0,8.0,3.4 \mathrm{~Hz}$, 1H, H-3), $6.84(\mathrm{dd}, J=5.0,2.0 \mathrm{~Hz}, 1 \mathrm{H}, \mathrm{H}-8), 7.01(\mathrm{~d}, J=2.4 \mathrm{~Hz}, 1 \mathrm{H}, \mathrm{ArH}), 7.12(\mathrm{td}, J=8.0,1.2 \mathrm{~Hz}$, $1 \mathrm{H}, \operatorname{ArH}), 7.20(\mathrm{td}, J=8.0,1.2 \mathrm{~Hz}, 1 \mathrm{H}, \operatorname{ArH}), 7.34(\mathrm{~d}, J=8.0 \mathrm{~Hz}, 1 \mathrm{H}, \operatorname{ArH}), 7.89(\mathrm{~d}, J=8.0 \mathrm{~Hz}, 1 \mathrm{H}$, ArH), 8.40 (s, 1H, NH ind); ${ }^{13} \mathrm{C}$ NMR (100.6 MHz, $\left.\mathrm{CDCl}_{3}\right) \delta 14.2\left(\mathrm{CH}_{3}\right), 22.6(\mathrm{C}-10), 25.5(\mathrm{C}-7), 26.3$ (C-11), $30.2\left(\mathrm{CH}_{2}\right.$-ind), $31.0(\mathrm{C}-6), 40.6$ (C-7a), 56.2 (C-3), $60.6\left(\mathrm{CH}_{2} \mathrm{CH}_{3}\right), 68.2(\mathrm{C}-2), 92.6(\mathrm{C}-11 \mathrm{a})$, 111.1 (CHAr), 111.6 (CAr), 119.2 (CHAr), 119.5 (CHAr), 122.0 (CHAr), 122.1 (CHAr), 127.5 (CAr), 129.6 (C-9), 136.2 (CAr), 137.7 (C-8), $166.5\left(\mathrm{CO}_{2}\right), 169.4$ (CO); HRMS (ESI-TOF) m/z: [M + H] $]^{+}$ Calcd for $\mathrm{C}_{23} \mathrm{H}_{27} \mathrm{~N}_{2} \mathrm{O}_{4}$ 395.1965; Found 395.1978. Anal. Calcd. for $\mathrm{C}_{23} \mathrm{H}_{26} \mathrm{~N}_{2} \mathrm{O}_{4} \cdot{ }^{1}{ }_{2} \mathrm{H}_{2} \mathrm{O}$ : C 68.47; $\mathrm{H}$ 6.74, N 6.94. Found: C 68.42; H 6.60; N 6.59. Lactam 7': $[\alpha]^{22}{ }_{D}-40.8\left(c 0.8, \mathrm{CHCl}_{3}\right) ; \mathrm{IR}\left(\right.$ film) $v\left(\mathrm{~cm}^{-}\right.$ 1) $1628(\mathrm{NCO}), 1706(\mathrm{COO}), 3297(\mathrm{NH}) ;{ }^{1} \mathrm{H} \mathrm{NMR}\left(400 \mathrm{MHz}, \mathrm{CDCl}_{3}, \mathrm{COSY}, g-\mathrm{HSQC}\right) \delta 1.28(\mathrm{t}, J=$ $7.2 \mathrm{~Hz}, 3 \mathrm{H}, \mathrm{CH}_{3}$ ), 1.53-1.63 (m, 2H, H-7, H-11), 2.02 (dd, $\left.J=14.0,6.0 \mathrm{~Hz}, 1 \mathrm{H}, \mathrm{H}-7\right), 2.11-2.20$ (m, 1H, H-11), 2.31-2.39 (m, 2H, H-7a, H-10), 2.48-2.51 (m, 3H, H-6, H-10), 2.76 (dd, J = 14.0, 9.6 Hz, 1H, $\mathrm{CH}_{2}$-ind), 3.79 (dd, $J=14.0,2.0 \mathrm{~Hz}, 1 \mathrm{H}, \mathrm{CH}_{2}$-ind), 3.97 (m, 2H, H-2), 4.18 (q, $J=7.2 \mathrm{~Hz}, 2 \mathrm{H}$, 
$\left.\mathrm{CH}_{2} \mathrm{CH}_{3}\right), 4.42(\mathrm{~m}, 1 \mathrm{H}, \mathrm{H}-3), 6.90(\mathrm{dd}, J=5.0,2.4 \mathrm{~Hz}, 1 \mathrm{H}, \mathrm{H}-8), 7.04(\mathrm{~d}, J=2.4 \mathrm{~Hz}, 1 \mathrm{H}, \mathrm{ArH}), 7.13$ $(\mathrm{td}, J=7.5,1.2 \mathrm{~Hz}, 1 \mathrm{H}, \mathrm{ArH}), 7.20(\mathrm{td}, J=7.5,1.2 \mathrm{~Hz}, 1 \mathrm{H}, \mathrm{ArH}), 7.36(\mathrm{~d}, J=7.5 \mathrm{~Hz}, 1 \mathrm{H}, \mathrm{ArH}), 7.80$ $(\mathrm{d}, J=7.5 \mathrm{~Hz}, 1 \mathrm{H}, \mathrm{ArH}), 8.07$ (s, $1 \mathrm{H}, \mathrm{NH}$ ind); ${ }^{13} \mathrm{C} \mathrm{NMR}\left(100.6 \mathrm{MHz}, \mathrm{CDCl}_{3}\right) \delta 14.2\left(\mathrm{CH}_{3}\right), 22.8(\mathrm{C}-$ 10), 25.7 (C-7), 26.3 (C-11), 26.6 ( $\mathrm{CH}_{2}$-ind), 30.1 (C-6), 39.8 (C-7a), 56.5 (C-3), $60.6\left(\mathrm{CH}_{2} \mathrm{CH}_{3}\right), 67.4$ (C-2), 92.7 (C-11a), 111.0 (CHAr), 112.4 (CAr), 119.3 (CHAr), 119.6 (CHAr), 122.2 (CHAr), 122.3 (CHAr), 127.7 (CAr), 129.2 (C-9), 136.1 (CAr), 138.8 (C-8), $166.6\left(\mathrm{CO}_{2}\right), 168.1$ (CO); HRMS (ESITOF) $m / z:[\mathrm{M}+\mathrm{H}]^{+}$Calcd for $\mathrm{C}_{23} \mathrm{H}_{27} \mathrm{~N}_{2} \mathrm{O}_{4}$ 395.1965; Found 395.1971.

\section{$(2 \mathrm{a} R, 6 \mathrm{a} S, 8 S, 14 \mathrm{~b} S)-4,8-B i s(h y d r o x y m e t h y l)-2,2 \mathrm{a}, 5,6,6 \mathrm{a}, 8,9,14 \mathrm{~b}-$-octahydro-1H-benz $[f]$ indolo[2,3-}

a]quinolizine (10): $\mathrm{POCl}_{3}(1.12 \mathrm{~mL}, 12.16 \mathrm{mmol})$ was added to a solution of lactam 7 (600 mg, 1.52 $\mathrm{mmol})$ in toluene $(20 \mathrm{~mL})$, and the solution was stirred at $100{ }^{\circ} \mathrm{C}$ for $1.5 \mathrm{~h}$. The solvent was evaporated, and dry methanol $(30 \mathrm{~mL})$ was added to the residue. $\mathrm{NaBH}_{4}(173 \mathrm{mg}, 4.57 \mathrm{mmol})$ was slowly added at 0 ${ }^{\circ} \mathrm{C}$ to the solution, and the mixture was stirred, allowing it to reach room temperature (about $1.5 \mathrm{~h}$ ). The reaction was quenched by addition of saturated aqueous $\mathrm{NaHCO}_{3}$. The methanol was evaporated, and the aqueous solution was extracted with $\mathrm{CH}_{2} \mathrm{Cl}_{2}$. The combined organic extracts were dried, filtered, and concentrated. $\mathrm{LiAlH}_{4}(866 \mathrm{mg}, 23 \mathrm{mmol})$ was added to a solution of the resulting residue in anhydrous THF $(25 \mathrm{~mL})$, and the mixture was stirred at reflux for $3 \mathrm{~h}$. The reaction was quenched at $0{ }^{\circ} \mathrm{C}$ with water $(866 \mu \mathrm{L})$, and $10 \%$ aqueous $\mathrm{NaOH}(866 \mu \mathrm{L})$ and then water $(2.5 \mathrm{~mL})$ were added. The resulting suspension was dried with $\mathrm{MgSO}_{4}$, filtered, and concentrated. Flash chromatography of the resulting oil (9:1 $\left.\mathrm{CH}_{2} \mathrm{Cl}_{2}-\mathrm{MeOH}\right)$ afforded pentacyclic compound $\mathbf{1 0}$ as a light yellow foam (308 $\mathrm{mg}, 60 \%$ ): IR (film) $v\left(\mathrm{~cm}^{-1}\right) 3402(\mathrm{OH}, \mathrm{NH}) ;{ }^{1} \mathrm{H}$ NMR (400 MHz, $\mathrm{CDCl}_{3}, \mathrm{COSY}, g$-HSQC) $\delta 1.14(\mathrm{qd}, J=12.8,4.8$ Hz, 1H, H-6), 1.40 (qd, $J=13.2,4.8 \mathrm{~Hz}, 1 \mathrm{H}, \mathrm{H}-2), 1.67$ (m, 2H, H-2, H-6), 1.78 (dd, $J=17.2,4.0 \mathrm{~Hz}$, 1H, H-5), 1.91 (m, 1H, H-5), 2.12 (m, 1H, H-1), 2.35 (m, 2H, H-1, H-2a), 2.80 (dd, J = 8.8, 1.6 Hz, 2H, H-9), 3.27 (dq, $J=12.8,2.4 \mathrm{~Hz}, 1 \mathrm{H}, \mathrm{H}-6 \mathrm{a}), 3.51$ (m, 1H, H-8), 3.82 (s, 2H, $\mathrm{CH}_{2} \mathrm{OH}$ ), 3.86 (dd, $J=11.6$, $\left.6.4 \mathrm{~Hz}, 1 \mathrm{H}, \mathrm{CH}_{2} \mathrm{OH}\right), 4.04\left(\mathrm{dd}, J=11.6,7.6 \mathrm{~Hz}, 1 \mathrm{H}, \mathrm{CH}_{2} \mathrm{OH}\right), 4.27$ (brs, $\left.1 \mathrm{H}, \mathrm{H}-14 \mathrm{~b}\right), 5.55$ (d, $J=4.0$ $\mathrm{Hz}, 1 \mathrm{H}, \mathrm{H}-3), 6.99$ (td, $J=7.6,0.8 \mathrm{~Hz}, 1 \mathrm{H}, \mathrm{ArH}), 7.06(\mathrm{td}, J=8.0,1.2 \mathrm{~Hz}, 1 \mathrm{H}, \mathrm{ArH}), 7.33(\mathrm{~d}, J=8.0$ $\mathrm{Hz}, 1 \mathrm{H}, \mathrm{ArH}), 7.37$ (d, $J=7.6 \mathrm{~Hz}, 1 \mathrm{H}, \mathrm{ArH}) ;{ }^{13} \mathrm{C} \mathrm{NMR}\left(100.6 \mathrm{MHz}, \mathrm{CDCl}_{3}\right) \delta 24.2$ (C-6), $25.1(\mathrm{C}-2)$, 
26.5 (C-9), 27.1 (C-1), 27.3 (C-5), 37.6 (C-2a), 53.8 (C-14b), 55.9 (C-6a), 62.9 (C-8), $64.3\left(\mathrm{CH}_{2} \mathrm{OH}\right)$, $66.7\left(\mathrm{CH}_{2} \mathrm{OH}\right), 108.5$ (CAr), 112.0 (CHAr), 118.3 (CHAr), 119.7 (CHAr), 121.7 (CHAr), 127.1 (C-3), 128.9 (CAr), 137.5 and 137.8 (2CAr, C-4); HRMS (ESI-TOF) $m / z:[\mathrm{M}+\mathrm{H}]^{+}$Calcd for $\mathrm{C}_{21} \mathrm{H}_{27} \mathrm{~N}_{2} \mathrm{O}_{2}$ 339.2067; Found 339.2064.

\section{$(9 S, 4 a S, 15 b R)-3,9-B i s(h y d r o x y m e t h y l)-1,2,4 a, 5,6,7,9,10-o c t a h y d r o b e n z[i]$ indolo[2,3-a $]$ quinolizine}

(11): Operating as in the above cyclization of lactam 7, from the minor isomer 7' $(180 \mathrm{mg}, 0.35 \mathrm{mmol})$ in toluene $(5.0 \mathrm{~mL})$ and $\mathrm{POCl}_{3}(257 \mu \mathrm{L}, 2.79 \mathrm{mmol})$, then $\mathrm{NaBH}_{4}(40 \mathrm{mg}, 1.05 \mathrm{mmol})$ in anhydrous methanol $(7.0 \mathrm{~mL})$, and finally $\mathrm{LiAlH}_{4}(200 \mathrm{mg}, 5.25 \mathrm{mmol})$ in THF $(6 \mathrm{~mL})$, compound $11(77 \mathrm{mg}$, $65 \%)$ was obtained after column chromatography $\left(9: 1 \mathrm{CH}_{2} \mathrm{Cl}_{2}-\mathrm{MeOH}\right): \mathrm{IR}$ (film) $v\left(\mathrm{~cm}^{-1}\right) 3405(\mathrm{OH}$, $\mathrm{NH}) ;{ }^{1} \mathrm{H}$ NMR (400 MHz, $\mathrm{CDCl}_{3}, \mathrm{COSY}, g$-HSQC) $\delta 1.40(\mathrm{dm}, J=12.4 \mathrm{~Hz}, 1 \mathrm{H}, \mathrm{H}-6), 1.51(\mathrm{dt}, J=$ 14.4, 4.4 Hz, 1H, H-5), 1.58 (dm, $J=12.4$ Hz, 1H, H-5), 1.60 (m, 1H, H-6), 1.79 (m, 1H, H-1), 2.01 $(\mathrm{dm}, J=16.0 \mathrm{~Hz}, 1 \mathrm{H}, \mathrm{H}-2), 2.25(\mathrm{dm}, J=16.0 \mathrm{~Hz}, 1 \mathrm{H}, \mathrm{H}-2), 2.30$ (ddd, $J=13.2,4.4,2.8 \mathrm{~Hz}, 1 \mathrm{H}, \mathrm{H}-1)$, $2.38(\mathrm{td}, J=12.0,2.4 \mathrm{~Hz}, 1 \mathrm{H}, \mathrm{H}-7), 2.51(\mathrm{dd}, J=15.6,4.0 \mathrm{~Hz}, 1 \mathrm{H}, \mathrm{H}-10), 2.63$ (dd, $J=15.6,10.4 \mathrm{~Hz}$, 1H, H-10), 2.73 (m, 1H, H-7), 2.84 (brs, 1H, H-4a), 3.64 (d, J = 5.2 Hz, 1H, CH $\left.H_{2} \mathrm{OH}\right), 3.75-3.79$ (m, 2H, $\left.\mathrm{H}-9, \mathrm{CH}_{2} \mathrm{OH}\right), 4.12\left(\mathrm{~s}, 2 \mathrm{H}, \mathrm{CH}_{2} \mathrm{OH}\right), 7.10(\mathrm{td}, J=7.2,0.8 \mathrm{~Hz}, 1 \mathrm{H}, \mathrm{ArH}), 7.16(\mathrm{td}, J=7.2,0.8 \mathrm{~Hz}, 1 \mathrm{H}$, ArH), $7.33(\mathrm{~d}, J=7.6 \mathrm{~Hz}, 1 \mathrm{H}, \mathrm{ArH}), 7.46(\mathrm{~d}, J=7.6 \mathrm{~Hz}, 1 \mathrm{H}, \mathrm{ArH}) ;{ }^{13} \mathrm{C} \mathrm{NMR}\left(100.6 \mathrm{MHz}, \mathrm{CDCl}_{3}\right) \delta$ 19.3 (C-10), 21.1 (C-6), 22.6 (C-2), 27.4 (C-5), 34.6 (C-1), 38.7 (C-4a, C-7), 54.0 (C-9), 56.5 (C-15b), $61.2\left(\mathrm{CH}_{2} \mathrm{OH}\right), 66.5\left(\mathrm{CH}_{2} \mathrm{OH}\right), 107.1$ (CAr), 110.9 (CHAr), 118.0 (CHAr), 119.5 (CHAr), 121.5 (CHAr), 125.0 (C-4), 127.4 (CAr), 135.4 (CAr), 137.9 (CAr), 138.5 (C-3); HRMS (ESI-TOF) m/z: [M + $\mathrm{H}]^{+}$Calcd for $\mathrm{C}_{21} \mathrm{H}_{27} \mathrm{~N}_{2} \mathrm{O}_{2}$ 339.2067; Found 339.2066.

Ethyl

$(3 S, 7 \mathrm{a} R, 11 \mathrm{a} R)-3-[1-(4-M e t h o x y b e n z y l)-3-i n d o l y l m e t h y l]-5-0 x 0-2,3,5,6,7,7 \mathrm{a}, 10,11-$ octahydrooxazolo[2,3-j]quinoline-9-carboxylate (12): Lactam 7 (195 mg, 0.49 mmol) was added at 0 ${ }^{\circ} \mathrm{C}$ under a nitrogen atmosphere to a suspension of $\mathrm{NaH}(60 \%$ dispersion in mineral oil, $18 \mathrm{mg}, 0.74$ mmol) in dry DMF (5 mL), and the resulting mixture was stirred for 30 minutes. 4-Methoxybenzyl chloride $(80 \mu \mathrm{l}, 0.59 \mathrm{mmol})$ was added, and the stirring was continued at $0{ }^{\circ} \mathrm{C}$ for $1 \mathrm{~h}$. After neutralization with saturated aqueous $\mathrm{NH}_{4} \mathrm{Cl}$, the mixture was extracted three times with $\mathrm{Et}_{2} \mathrm{O}$. The 
combined organic extracts were washed with brine, dried, filtered, and concentrated. Flash chromatography of the residue (4:1 hexane-EtOAc) provided lactam 12 (170 mg, 67\%): IR (film) $v\left(\mathrm{~cm}^{-1}\right) 1643(\mathrm{NCO}), 1707(\mathrm{COO}) ;{ }^{1} \mathrm{H}$ NMR (400 MHz, CDCl 3 , COSY, $g$-HSQC) $\delta 1.28(\mathrm{t}, J=6.8 \mathrm{~Hz}$, $3 \mathrm{H}, \mathrm{CH}_{3}$ ), 1.53-1.57 (m, 2H, H-7, H-11), 1.84 (dd, $\left.J=14.0,5.6 \mathrm{~Hz}, 1 \mathrm{H}, \mathrm{H}-11\right), 2.04-2.10$ (m, 1H, H-7), 2.25-2.29 (m, 1H, H-7a), 2.29-2.34 (m, 1H, H-10), 2.39 (m, 1H, H-10), 2.46 (ddd, J = 18.8, 11.6, 7.2 Hz, 1H, H-6), 2.62 (dd, $J=18.8,5.2 \mathrm{~Hz}, 1 \mathrm{H}, \mathrm{H}-6$ ), 2.93 (dd, $J=14.4,4.0 \mathrm{~Hz}, 1 \mathrm{H}, \mathrm{CH}_{2}$-ind), 3.56 (dd, $J$ $=14.4,3.2 \mathrm{~Hz}, 1 \mathrm{H}, \mathrm{CH}_{2}$-ind), $3.77\left(\mathrm{~s}, 3 \mathrm{H}, \mathrm{CH}_{3} \mathrm{O}\right), 3.79\left(\mathrm{t}, J=8.8 \mathrm{~Hz}, 1 \mathrm{H}, \mathrm{CH}_{2} \mathrm{O}\right), 4.00(\mathrm{t}, J=8.8 \mathrm{~Hz}$, $\left.1 \mathrm{H}, \mathrm{CH}_{2} \mathrm{O}\right), 4.18\left(\mathrm{q}, J=6.8 \mathrm{~Hz}, 2 \mathrm{H}, \mathrm{CH}_{2} \mathrm{CH}_{3}\right), 4.64(\mathrm{ddd}, J=11.2,8.0,3.2 \mathrm{~Hz}, 1 \mathrm{H}, \mathrm{H}-3), 5.20(\mathrm{~s}, 2 \mathrm{H}$, $\left.\mathrm{NCH}_{2}\right), 6.83(\mathrm{~d}, J=8.4 \mathrm{~Hz}, 3 \mathrm{H}, \mathrm{H}-8, \mathrm{ArH}), 6.93$ (s, 1H, H-2 ind), 7.06 (d, J = 8.4 Hz, 2H, ArH), 7.12 (t, $J=7.2 \mathrm{~Hz}, 1 \mathrm{H}, \operatorname{ArH}), 7.19(\mathrm{t}, J=7.2 \mathrm{~Hz}, 1 \mathrm{H}, \mathrm{ArH}), 7.28(\mathrm{~d}, J=8.0 \mathrm{~Hz}, 1 \mathrm{H}, \operatorname{ArH}), 7.78(\mathrm{~d}, J=8.0 \mathrm{~Hz}$, 1H, ArH); ${ }^{13} \mathrm{C}$ NMR $\left(100.6 \mathrm{MHz}, \mathrm{CDCl}_{3}\right) \delta 14.2\left(\mathrm{CH}_{3}\right), 22.6(\mathrm{C}-10), 25.6(\mathrm{C}-7), 26.2(\mathrm{C}-11), 29.9$ $\left(\mathrm{CH}_{2}\right.$-ind), $30.9(\mathrm{C}-6), 40.6(\mathrm{C}-7 \mathrm{a}), 49.3\left(\mathrm{NCH}_{2}\right), 55.2\left(\mathrm{CH}_{3} \mathrm{O}\right), 56.3(\mathrm{C}-3), 60.5\left(\mathrm{CH}_{3} \mathrm{CH}_{2}\right), 68.0$ ( $\left.\mathrm{CH}_{2} \mathrm{O}\right), 92.6$ (C-11a), 109.6 (CHAr), 110.8 (CAr), 114.1 (CHAr), 119.3 (CHAr), 119.5 (CHAr), 121.9 (CHAr), 126.0 (CHAr), 128.2 (CHAr), 129.4 (CAr), 129.6 (CAr), 130.0 (C-9), 136.5 (CAr), 137.7 (C8), 159.1 (CAr), $166.5\left(\mathrm{CO}_{2}\right), 169.3$ (CO); HRMS (ESI-TOF) $m / z:[\mathrm{M}+\mathrm{H}]^{+}$Calcd for $\mathrm{C}_{31} \mathrm{H}_{35} \mathrm{~N}_{2} \mathrm{O}_{5}$ 515.2540; Found 515.2545.

\section{(2aR,6aS,8S,14bS)-4,8-Bis(hydroxymethyl)-14-(4-methoxybenzyl)-2,2a,5,6,6a,8,9,14b-octahydro-}

$1 H$-benz $[f]$ indolo $[2,3-a]$ quinolizine (13): Operating as in the preparation of compound 10, from lactam $12(2.04 \mathrm{~g}, 4.66 \mathrm{mmol})$ in toluene $(58 \mathrm{~mL})$ and $\mathrm{POCl}_{3}(3.4 \mathrm{~mL}, 37.3 \mathrm{mmol})$, then $\mathrm{NaBH}_{4}(540 \mathrm{mg}, 14$ mmol) in anhydrous methanol $(92 \mathrm{~mL})$, and finally $\mathrm{LiAlH}_{4}(1.77 \mathrm{~g}, 46.6 \mathrm{mmol})$ in THF $(90 \mathrm{~mL})$, pentacyclic diol $13(1.45 \mathrm{~g}, 68 \%)$ was obtained after purification by column chromatography (99:1 $\mathrm{CH}_{2} \mathrm{Cl}_{2}-\mathrm{MeOH}$ ): mp: $119-122{ }^{\circ} \mathrm{C} ;[\alpha]^{22}{ }_{\mathrm{D}}-34.0$ (c 0.4, $\mathrm{CHCl}_{3}$ ); IR (film) v (cm $\left.{ }^{-1}\right) 3428(\mathrm{OH}) ;{ }^{1} \mathrm{H} \mathrm{NMR}$ (400 MHz, CDCl 3 , COSY, g-HSQC) $\delta 1.26-1.41$ (m, 2H, H-2, H-6), 1.60-1.70 (m, 2H, H-2, H-6), 1.81 (dd, $J=14.4,3.2$ Hz, 1H, H-5), 1.87-2.02 (m, 2H, H-1, H-5), 2.19-2.25 (m, 1H, H-1), 2.39 (m, 1H, H2a), 2.78-2.82 (m, 2H, H-9), 3.31 (ddd, $J=12.0,5.2,3.2 \mathrm{~Hz}, 1 \mathrm{H}, \mathrm{H}-6 \mathrm{a}), 3.53$ (tt, $J=8.8,6.4 \mathrm{~Hz}, 1 \mathrm{H}, \mathrm{H}-$ 8), $3.75\left(\mathrm{~s}, 3 \mathrm{H}, \mathrm{CH}_{3} \mathrm{O}\right), 3.75-3.81\left(\mathrm{dd}, J=11.2,5.2 \mathrm{~Hz}, 1 \mathrm{H}, \mathrm{CH}_{2} \mathrm{OH}\right), 3.90\left(\mathrm{~s}, 2 \mathrm{H}, \mathrm{CH}_{2} \mathrm{OH}\right), 3.87-3.95$ 
$\left(\mathrm{dd}, J=12.0,5.2 \mathrm{~Hz}, 1 \mathrm{H}, \mathrm{CH}_{2} \mathrm{OH}\right), 4.22(\mathrm{t}, J=5.2 \mathrm{~Hz}, 1 \mathrm{H}, \mathrm{H}-14 \mathrm{~b}), 5.25\left(\mathrm{~d}, J=17.2 \mathrm{~Hz}, 1 \mathrm{H}, \mathrm{NCH}_{2}\right)$, $5.43\left(\mathrm{~d}, J=17.2 \mathrm{~Hz}, 1 \mathrm{H}, \mathrm{NCH}_{2}\right), 5.48(\mathrm{~d}, J=3.2 \mathrm{~Hz}, 1 \mathrm{H}, \mathrm{H}-3), 6.78(\mathrm{~d}, J=8.4 \mathrm{~Hz}, 2 \mathrm{H}, \mathrm{ArH}), 6.83(\mathrm{~d}, J$ $=8.4 \mathrm{~Hz}, 2 \mathrm{H}, \mathrm{ArH}), 7.11(\mathrm{td}, J=7.2,1.2 \mathrm{~Hz}, 1 \mathrm{H}, \mathrm{ArH}), 7.15(\mathrm{td}, J=6.8,1.2 \mathrm{~Hz}, 1 \mathrm{H}, \operatorname{ArH}), 7.23(\mathrm{~d}, J=$ $8.0 \mathrm{~Hz}, 1 \mathrm{H}, \mathrm{ArH}), 7.47(\mathrm{~d}, J=6.8 \mathrm{~Hz}, 1 \mathrm{H}, \mathrm{ArH}) ;{ }^{13} \mathrm{C} \mathrm{NMR}\left(100.6 \mathrm{MHz}, \mathrm{CDCl}_{3}\right) \delta 24.8(\mathrm{C}-6), 25.0(\mathrm{C}-$ 2), 25.2 (C-9), 26.2 (C-5), 27.1 (C-1), $36.2(\mathrm{C}-2 \mathrm{a}), 47.5\left(\mathrm{NCH}_{2}\right), 53.0(\mathrm{C}-6 \mathrm{a}), 54.5$ (C-14b), 55.2 $\left(\mathrm{CH}_{3} \mathrm{O}\right), 61.0(\mathrm{C}-8), 62.5\left(\mathrm{CH}_{2} \mathrm{OH}\right), 66.6\left(\mathrm{CH}_{2} \mathrm{OH}\right), 109.3(\mathrm{CHAr}), 110.0(\mathrm{CAr}), 114.2(\mathrm{CHAr}), 117.8$ (CHAr), 119.4 (CHAr), 121.5 (CHAr), 126.4 (C-3), 126.6 (CHAr), 126.9, 130.1 and 136.9 (C-4, 2CAr), 137.9 (CAr), 158.7 (CAr); HRMS (ESI-TOF) $m / z:[\mathrm{M}+\mathrm{H}]^{+}$Calcd for $\mathrm{C}_{29} \mathrm{H}_{35} \mathrm{~N}_{2} \mathrm{O}_{3}$ 459.2642; Found 459.2628 .

\section{(2aR,6aS,8S,14bS)-4-[(tert-Butyldiphenylsilyloxy)methyl]-8-(hydroxymethyl)-14-(4-}

methoxybenzyl)-2,2a,5,6,6a,8,9,14b-octahydro-1H-benz[f]indolo[2,3-a $]$ quinolizine (14): Imidazole (32 mg, $0.48 \mathrm{mmol}$ ) and tert-butyldiphenylsilyl chloride $\left(191 \mu \mathrm{l}, 0.72 \mathrm{mmol}\right.$ ) were added at $0{ }^{\circ} \mathrm{C}$ to a solution of diol 13 (220 mg, $0.48 \mathrm{mmol})$ in $\mathrm{CH}_{2} \mathrm{Cl}_{2}(25 \mathrm{~mL})$. The solution was stirred for 15 minutes at this temperature. Then saturated aqueous $\mathrm{NH}_{4} \mathrm{Cl}$ was added, and the mixture was extracted with $\mathrm{CH}_{2} \mathrm{Cl}_{2}$. The combined organic extracts were dried, filtered, and concentrated. Flash chromatography (99:1 $\left.\mathrm{CH}_{2} \mathrm{Cl}_{2}-\mathrm{MeOH}\right)$ of the residue afforded alcohol 14 as a yellow foam $(254 \mathrm{mg}, 76 \%)$ : IR (film) $v\left(\mathrm{~cm}^{-1}\right)$ $3428(\mathrm{OH}), 1109$ (OSi); ${ }^{1} \mathrm{H}$ NMR (400 MHz, CDCl 3 , COSY, g-HSQC) $\delta 1.02$ [s, 9H, C(CH3) 3 , $1.26-$ 1.37 (m, 2H, H-2, H-6), 1.60-1.66 (m, 2H, H-2, H-6), 1.80-1.83 (m, 2H, H-5), 1.93-2.04 (m, 1H, H-1), 2.25-2.29 (m, 1H, H-1), 2.39 (m, 1H, H-2a), 2.79-2.82 (m, 2H, H-9), 3.31 (dm, J = 12.0 Hz, 1H, H-6a), 3.53-3.57 (m, 1H, H-8), $3.73\left(\mathrm{~s}, 3 \mathrm{H}, \mathrm{CH}_{3} \mathrm{O}\right), 3.81\left(\mathrm{dd}, J=10.8,4.8 \mathrm{~Hz}, 1 \mathrm{H}, \mathrm{CH}_{2} \mathrm{OH}\right), 3.94(\mathrm{~s}, 2 \mathrm{H}$, $\left.\mathrm{CH}_{2} \mathrm{OSi}\right), 3.91-3.96\left(\mathrm{~m}, 1 \mathrm{H}, \mathrm{CH}_{2} \mathrm{OH}\right), 4.26(\mathrm{~m}, 1 \mathrm{H}, \mathrm{H}-14 \mathrm{~b}), 5.27$ (d, $\left.J=17.6 \mathrm{~Hz}, 1 \mathrm{H}, \mathrm{NCH}_{2}\right), 5.45$ (d, $J$ $\left.=17.6 \mathrm{~Hz}, 1 \mathrm{H}, \mathrm{NCH}_{2}\right), 5.48(\mathrm{~m}, 1 \mathrm{H}, \mathrm{H}-3), 6.79(\mathrm{~d}, J=8.4 \mathrm{~Hz}, 2 \mathrm{H}, \mathrm{ArH}), 6.84(\mathrm{~d}, J=8.4 \mathrm{~Hz}, 2 \mathrm{H}, \mathrm{ArH})$, $7.11(\mathrm{td}, J=7.6,1.2 \mathrm{~Hz}, 1 \mathrm{H}, \mathrm{ArH}), 7.16(\mathrm{td}, J=8.0,1.2 \mathrm{~Hz}, 1 \mathrm{H}, \mathrm{ArH}), 7.24(\mathrm{~d}, J=7.2 \mathrm{~Hz}, 1 \mathrm{H}, \operatorname{ArH})$, 7.31-7.41 (m, 6H, ArH), 7.47 (d, $J=6.8 \mathrm{~Hz}, 1 \mathrm{H}, \mathrm{ArH}), 7.60-7.64(\mathrm{~m}, 4 \mathrm{H}, \mathrm{ArH}) ;{ }^{13} \mathrm{C} \mathrm{NMR}(100.6 \mathrm{MHz}$, $\left.\mathrm{CDCl}_{3}\right) \delta 19.2\left[C\left(\mathrm{CH}_{3}\right)_{3}\right], 24.7$ and $24.8(\mathrm{C}-6, \mathrm{C}-2), 25.2(\mathrm{C}-9), 26.1(\mathrm{C}-5), 26.8\left[\mathrm{C}\left(\mathrm{CH}_{3}\right)_{3}\right], 27.1(\mathrm{C}-1)$, $36.3(\mathrm{C}-2 \mathrm{a}), 47.5\left(\mathrm{NCH}_{2}\right), 53.0(\mathrm{C}-6 \mathrm{a}), 54.6(\mathrm{C}-14 \mathrm{~b}), 55.2\left(\mathrm{CH}_{3} \mathrm{O}\right), 61.3(\mathrm{C}-8), 62.4\left(\mathrm{CH}_{2} \mathrm{OH}\right), 67.1$ 
( $\mathrm{CH}_{2} \mathrm{OSi}$ ), 109.3 (CHAr), 110.0 (CAr), 114.2 (CHAr), 117.8 (CHAr), 119.4 (CHAr), 121.5 (CHAr), 125.1 (C-3), 126.6 (CHAr), 126.9 (CAr), 130.1 (CAr), 133.7 (CAr), 133.7 (CAr), 136.2 (C-4, CAr), 137.9 (CAr), 158.7 (CAr); HRMS (ESI-TOF) $m / z:[\mathrm{M}+\mathrm{H}]^{+}$Calcd for $\mathrm{C}_{45} \mathrm{H}_{53} \mathrm{~N}_{2} \mathrm{O}_{3} \mathrm{Si}$ 697.3820; Found 697.3807.

\section{(2aR,6aS,8S,14bS)-4-[(tert-Butyldiphenylsilyloxy)methyl]-8-formyl-14-(4-methoxybenzyl)-}

2,2a,5,6,6a,8,9,14b-octahydro-1H-benz[f]indolo[2,3-a $]$ quinolizine (15): $4 \AA$ Powdered sieves (376 $\mathrm{mg}$ ) and NMO (115 mg, $0.96 \mathrm{mmol})$ were added at room temperature under an inert atmosphere to a solution of alcohol 14 (190 mg, $0.27 \mathrm{mmol})$ in $\mathrm{CH}_{3} \mathrm{CN}$ (6 mL). Tetrapropylammonium perruthenate (19 $\mathrm{mg}, 0.054 \mathrm{mmol}$ ) was then added in one portion, and the resulting mixture was stirred at room temperature for 30 minutes. The solvent was evaporated, and the dark residue was dissolved in $\mathrm{CH}_{2} \mathrm{Cl}_{2}$. The solution was filtered through a short pad of silica using $\mathrm{CH}_{2} \mathrm{Cl}_{2}$ as the eluent. The filtrate was concentrated to give aldehyde 15 (95 mg, 50\%), which was used in the next step without purification: IR (film) $v\left(\mathrm{~cm}^{-1}\right) 1656(\mathrm{CHO}), 1110(\mathrm{OSi}) ;{ }^{1} \mathrm{H}$ NMR (400 MHz, CDCl $\left.3, \mathrm{COSY}, g-\mathrm{HSQC}\right) \delta 1.03$ [s, 9H, $\left.\mathrm{C}\left(\mathrm{CH}_{3}\right)_{3}\right], 1.49$ (qd, $\left.J=12.8,4.0 \mathrm{~Hz}, 1 \mathrm{H}, \mathrm{H}-1\right), 1.65-1.79$ (m, 3H, H-2, H-6), $1.83(\mathrm{dm}, J=12.8 \mathrm{~Hz}, 1 \mathrm{H}$, H-1), 1.93-1.96 (m, 2H, H-5), 2.14 (m, 1H, H-6), 2.47 (s, 1H, H-2a), 3.04 (ddd, J = 15.2, 5.6, 2.0 Hz, 1H, H-9), 3.23 (d, $J=15.2 \mathrm{~Hz}, 1 \mathrm{H}, \mathrm{H}-9), 3.73$ (m, 1H, H-6a), 3.74 (s, 3H, $\mathrm{CH}_{3} \mathrm{O}$ ), 3.97 (m, 1H, H-8), 4.08 (s, 2H, $\left.\mathrm{CH}_{2} \mathrm{OSi}\right), 4.18$ (d, $\left.J=10.8 \mathrm{~Hz}, 1 \mathrm{H}, \mathrm{H}-14 \mathrm{~b}\right), 5.14$ (d, $\left.J=16.8 \mathrm{~Hz}, 1 \mathrm{H}, \mathrm{NCH}_{2}\right), 5.23(\mathrm{~d}, J=$ $\left.16.8 \mathrm{~Hz}, 1 \mathrm{H}, \mathrm{NCH}_{2}\right), 5.27$ (s, 1H, H-3), 6.78 (d, J=8.8 Hz, 2H, ArH), 6.87 (d, J=8.8 Hz, 2H, ArH), $7.03(\mathrm{~m}, 1 \mathrm{H}, \mathrm{ArH})$, 7.06-7.09 (m, 2H, ArH), 7.24-7.34 (m, 6H, ArH), 7.51 (m, 1H, ArH), 7.64-7.68 (m, 4H, $\mathrm{ArH}), 9.66$ (s, 1H, CHO); ${ }^{13} \mathrm{C} \mathrm{NMR}\left(100.6 \mathrm{MHz}, \mathrm{CDCl}_{3}\right) \delta 19.2\left[C\left(\mathrm{CH}_{3}\right)_{3}\right], 21.3(\mathrm{C}-5), 23.3(\mathrm{C}-9)$, $26.8\left[\mathrm{C}\left(\mathrm{CH}_{3}\right)_{3}, \mathrm{C}-6\right], 29.4(\mathrm{C}-1), 30.6(\mathrm{C}-2), 35.9(\mathrm{C}-2 \mathrm{a}), 47.2\left(\mathrm{NCH}_{2}\right), 55.2\left(\mathrm{CH}_{3} \mathrm{O}\right), 55.3(\mathrm{C}-6 \mathrm{a}), 55.6$ (C-14b), 59.8 (C-8), 67.6 ( $\left.\mathrm{CH}_{2} \mathrm{OSi}\right), 107.1$ (CAr), 110.0 (CHAr), 114.1 (CHAr), 117.9 (CHAr), 119.4 (CHAr), 121.4 (CHAr), 124.3 (C-3), 126.7 (CAr), 127.0 (CHAr), 127.5 (CHAr), 129.5 (CHAr), 135.4 (CAr), 135.5 (CAr), 137.1 (CAr), 137.8 (C-4), 137.9 (CAr), 158.7 (CAr), 205.6 (CHO); HRMS (ESITOF) $m / z:[\mathrm{M}+\mathrm{H}]^{+}$Calcd for $\mathrm{C}_{45} \mathrm{H}_{51} \mathrm{~N}_{2} \mathrm{O}_{3} \mathrm{Si}$ 695.3663; Found 695.3636. 
$(2 \mathrm{a} R, 6 \mathrm{a} S, 8 S, 14 \mathrm{bS})-4-[($ tert-Butyldiphenylsilyloxy)methyl]-8-[(hydroxyimino)methyl]-14-(4-

methoxybenzyl)-2,2a,5,6,6a,8,9,14b-octahydro-1H-benz[f]indolo[2,3-a]quinolizine

(16):

$\mathrm{NH}_{2} \mathrm{OH} \cdot \mathrm{HCl}$ (42 mg, $\left.0.59 \mathrm{mmol}\right)$ was added to a solution of aldehyde 15 (75 mg, $\left.0.11 \mathrm{mmol}\right)$ in pyridine $(250 \mu \mathrm{L})$ and ethanol $(250 \mu \mathrm{L})$. The mixture was heated at reflux for $2 \mathrm{~h}$, and then the solvent was removed under reduced pressure. $0.2 \mathrm{~N}$ Aqueous $\mathrm{H}_{2} \mathrm{SO}_{4}(2 \mathrm{~mL})$ was added, and the mixture was stirred for 10 minutes and extracted with EtOAc. The organic extracts were washed with $2 \mathrm{~N}$ aqueous $\mathrm{NaOH}$, dried, filtered, and concentrated to afford oximes 16 (44 mg, 56\%): Major isomer: IR (film) $v\left(\mathrm{~cm}^{-1}\right) 1110(\mathrm{OSi}) ;{ }^{1} \mathrm{H}$ NMR $\left.\left(400 \mathrm{MHz}, \mathrm{CDCl}_{3}, \mathrm{COSY}, g \text {-HSQC) } \delta 1.02 \text { [s, 9H, C(CH}\right)_{3}\right], 1.48-1.52$ (m, 2H, H-2, H-5), 1.64-1.67 (m, 3H, H-1, H-2, H-6), 1.91 (m, 1H, H-5), 2.00 (m, 1H, H-1), 2.32 (d, $J=$ 14.0 Hz, 1H, H-6), 2.46 (m, 1H, H-2a), 2.79 (d, $J=15.2$ Hz, 1H, H-9), 3.06 (brs, 1H, H-6a), 3.16 (ddd, $J$ $=15.2,5.2,2.0 \mathrm{~Hz}, 1 \mathrm{H}, \mathrm{H}-9), 3.68(\mathrm{~d}, J=10.4 \mathrm{~Hz}, 1 \mathrm{H}, \mathrm{H}-14 \mathrm{~b}), 3.75\left(\mathrm{~s}, 3 \mathrm{H}, \mathrm{CH}_{3} \mathrm{O}\right), 4.08(\mathrm{~s}, 2 \mathrm{H}$, $\left.\mathrm{CH}_{2} \mathrm{OSi}\right), 4.15(\mathrm{~m}, 1 \mathrm{H}, \mathrm{H}-8), 5.17\left(\mathrm{~d}, J=17.2 \mathrm{~Hz}, 1 \mathrm{H}, \mathrm{NCH}_{2}\right), 5.29(\mathrm{~m}, 1 \mathrm{H}, \mathrm{H}-3), 5.30$ (d, J = 17.2 Hz, $\left.1 \mathrm{H}, \mathrm{NCH}_{2}\right), 6.80(\mathrm{~d}, J=8.4 \mathrm{~Hz}, 2 \mathrm{H}, \mathrm{ArH}), 6.88(\mathrm{~d}, J=8.4 \mathrm{~Hz}, 2 \mathrm{H}, \mathrm{ArH}), 7.09(\mathrm{~d}, J=4.8 \mathrm{~Hz}, 2 \mathrm{H}, \operatorname{ArH})$, 7.28-7.36 (m, 7H, ArH), 7.33 (m, 1H, CHNOH), 7.48 (m, 1H, ArH), 7.64-7.68 (m, 4H, ArH); ${ }^{13} \mathrm{C}$ NMR $\left(100.6 \mathrm{MHz}, \mathrm{CDCl}_{3}\right) \delta 19.3\left[C\left(\mathrm{CH}_{3}\right)_{3}\right], 21.2(\mathrm{C}-2), 26.6(\mathrm{C}-9), 26.8\left[\mathrm{C}\left(\mathrm{CH}_{3}\right)_{3}, \mathrm{C}-6\right], 29.7(\mathrm{C}-1), 29.7(\mathrm{C}-$ 5), $36.0(\mathrm{C}-2 \mathrm{a}), 47.4\left(\mathrm{NCH}_{2}\right), 50.0(\mathrm{C}-8), 55.2\left(\mathrm{CH}_{3} \mathrm{O}\right), 55.4(\mathrm{C}-6 \mathrm{a}), 55.6(\mathrm{C}-14 \mathrm{~b}), 67.5\left(\mathrm{CH}_{2} \mathrm{OSi}\right), 107.0$ (CAr), 109.8 (CHAr), 114.1 (CHAr), 118.0 (CHAr), 119.3 (CHAr), 121.4 (CHAr), 124.2 (C-3), 127.0 (CHAr), 127.2 (CHAr), 127.5 (CHAr), 127.6 (CHAr), 129.5 (CHAr), 129.7 (CAr), 133.9 (CAr), 134.0 (CAr), 135.4 (CHAr), 135.5 (CHAr), 136.7 (CAr), 137.5 (C-4), 138.3 (CAr), 151.2 (CHNOH), 158.8 (CAr); HRMS (ESI-TOF) $m / z$ : [M + H] ${ }^{+}$Calcd for $\mathrm{C}_{45} \mathrm{H}_{52} \mathrm{~N}_{3} \mathrm{O}_{3} \mathrm{Si}$ 710.3772; Found 710.3762. Minor isomer: ${ }^{1} \mathrm{H} \mathrm{NMR}\left(400 \mathrm{MHz}, \mathrm{CDCl}_{3}, \mathrm{COSY}, g\right.$-HSQC, selected resonances) $\delta 1.02\left[\mathrm{~s}, 9 \mathrm{H}, \mathrm{C}\left(\mathrm{CH}_{3}\right)_{3}\right]$, 2.35 (dm, $J=14.0 \mathrm{~Hz}, 1 \mathrm{H}, \mathrm{H}-6), 2.43$ (m, 1H, H-2a), 2.89 (d, J = 15.2 Hz, 1H, H-9), 2.96 (brs, 1H, H6a), 3.10 (m, 1H, H-9), 3.65 (d, J = $10.4 \mathrm{~Hz}, 1 \mathrm{H}, \mathrm{H}-14 \mathrm{~b}), 3.75$ (s, 3H, $\mathrm{CH}_{3} \mathrm{O}$ ), 4.08 (s, 2H, $\mathrm{CH}_{2} \mathrm{OSi}$ ), $4.82(\mathrm{~m}, 1 \mathrm{H}, \mathrm{H}-8), 5.18$ (d, $\left.J=17.2 \mathrm{~Hz}, 1 \mathrm{H}, \mathrm{NCH}_{2}\right), 5.27(\mathrm{~m}, 1 \mathrm{H}, \mathrm{H}-3), 5.33$ (d, $J=17.2 \mathrm{~Hz}, 1 \mathrm{H}$, $\left.\mathrm{NCH}_{2}\right), 6.76(\mathrm{~m}, 1 \mathrm{H}, C H \mathrm{NOH}), 6.78(\mathrm{~d}, J=8.4 \mathrm{~Hz}, 2 \mathrm{H}, \mathrm{ArH}), 6.87(\mathrm{~d}, J=8.4 \mathrm{~Hz}, 2 \mathrm{H}, \mathrm{ArH}), 7.09(\mathrm{~s}$, 1H, ArH), 7.28-7.36 (m, 7H, ArH), 7.48 (m, 1H, ArH), 7.64-7.68 (m, 4H, ArH). 
$(2 \mathrm{a} R, 6 \mathrm{a} S, 14 \mathrm{~b} S)-4-[($ tert-Butyldiphenylsilyloxy)methyl]-14-(4-methoxybenzyl)-2,2a,5,6,6a,8,9,14b-

octahydro-1H-benz[f]indolo[2,3-a]quinolizine (17): First step: Burgess reagent was added in three portions ( $3 \times 30 \mathrm{mg})$ to a solution of oximes $16(88 \mathrm{mg}, 0.12 \mathrm{mmol})$ in $\mathrm{CH}_{2} \mathrm{Cl}_{2}(1.25 \mathrm{~mL})$ over a period of 2 hours. The resulting solution was stirred at room temperature for 2 hours. Water was then added, and the mixture was extracted with $\mathrm{CH}_{2} \mathrm{Cl}_{2}$. The combined organic extracts were dried, filtered, and concentrated to afford the corresponding cyano derivative, which was used in the next step without purification. Second step: $\mathrm{AcOH}(17 \mu \mathrm{L})$ was added to a solution of $\mathrm{NaBH}_{3} \mathrm{CN}(28 \mathrm{mg}, 0.45 \mathrm{mmol})$ in $\mathrm{CH}_{3} \mathrm{CN}(120 \mu \mathrm{L})$, and the solution was stirred at room temperature for 30 minutes. Then a solution of the above cyano compound $(88 \mathrm{mg}, 0.075 \mathrm{mmol})$ in $\mathrm{CH}_{3} \mathrm{CN}(115 \mu \mathrm{L})$ was added, and the resulting mixture was stirred at room temperature for $9 \mathrm{~h} . \mathrm{CH}_{2} \mathrm{Cl}_{2}$ and $4 \mathrm{~N}$ aqueous $\mathrm{NaOH}$ were then added, and the mixture was extracted with $\mathrm{CH}_{2} \mathrm{Cl}_{2}$. The organic extracts were washed with brine, dried, filtered, and concentrated. Flash chromatography of the residue (9:1 hexane-EtOAc) afforded compound 17 (35 mg, 70\%, two steps): IR (film) v ( $\left.\mathrm{cm}^{-1}\right) 1111(\mathrm{OSi}) ;{ }^{1} \mathrm{H} \mathrm{NMR}\left(400 \mathrm{MHz}, \mathrm{CDCl}_{3}, \mathrm{COSY}, g\right.$-HSQC) $\delta 1.06$ [s, 9H, $\left.\mathrm{C}\left(\mathrm{CH}_{3}\right)_{3}\right], 1.56$ (td, $\left.J=11.6,4.8 \mathrm{~Hz}, 1 \mathrm{H}, \mathrm{H}-1\right), 1.62-1.72$ (m, 3H, H-2, H-6), 1.88-1.92 (m, 2H, H-1, H-5), 2.11 (t, $J=14.8 \mathrm{~Hz}, 1 \mathrm{H}, \mathrm{H}-5$ ), 2.22-2.28 (m, 2H, H-6, H-8), 2.48 (brs, 1H, H-2a), 2.70-2.76 (m, 2H, H-6a, H-9), 2.88 (m, 1H, H-9), 3.40-3.47 (m, 2H, H-8, H-14b), 3.77 (s, 3H, $\mathrm{CH}_{3} \mathrm{O}$ ), 4.11 (s, 2H, $\left.\mathrm{CH}_{2} \mathrm{OSi}\right), 5.18\left(\mathrm{~d}, J=17.2 \mathrm{~Hz}, 1 \mathrm{H}, \mathrm{NCH}_{2}\right), 5.30\left(\mathrm{~d}, J=17.2 \mathrm{~Hz}, 1 \mathrm{H}, \mathrm{NCH}_{2}\right), 5.33(\mathrm{~m}, 1 \mathrm{H}, \mathrm{H}-3), 6.80$ $(\mathrm{d}, J=8.8 \mathrm{~Hz}, 2 \mathrm{H}, \mathrm{ArH}), 6.88(\mathrm{~d}, J=8.8 \mathrm{~Hz}, 2 \mathrm{H}, \mathrm{ArH}), 7.06-7.10(\mathrm{~m}, 3 \mathrm{H}, \mathrm{ArH}), 7.29-7.37(\mathrm{~m}, 7 \mathrm{H}$, ArH), 7.51-7.53 (m, 1H, ArH), $7.48(\mathrm{~m}, 1 \mathrm{H}, \mathrm{ArH}), 7.67-7.70(\mathrm{~m}, 2 \mathrm{H}, \mathrm{ArH}) ;{ }^{13} \mathrm{C} \mathrm{NMR}(100.6 \mathrm{MHz}$, $\left.\mathrm{CDCl}_{3}\right) \delta 19.3\left[C\left(\mathrm{CH}_{3}\right)_{3}\right], 21.3(\mathrm{C}-5), 23.1(\mathrm{C}-9), 26.8\left[\mathrm{C}\left(\mathrm{CH}_{3}\right)_{3}\right], 27.1(\mathrm{C}-6), 28.5(\mathrm{C}-1), 30.2(\mathrm{C}-2)$, $36.0(\mathrm{C}-2 \mathrm{a}), 46.3(\mathrm{C}-8), 47.6\left(\mathrm{NCH}_{2}\right), 55.2\left(\mathrm{CH}_{3} \mathrm{O}\right), 58.6(\mathrm{C}-6 \mathrm{a}), 59.9(\mathrm{C}-14 \mathrm{~b}), 67.5\left(\mathrm{CH}_{2} \mathrm{OSi}\right), 109.7$ (CHAr), 114.0 (CHAr), 118.0 (CHAr), 119.2 (CHAr), 121.1 (CHAr), 124.6 (C-3), 127.0 (CHAr), 127.1 (CHAr), 127.5 (CHAr), 129.0 (CAr), 129.5 (CHAr), 129.5 (CHAr), 129.9 (CAr), 133.9 (CAr), 134.0 (CAr), 135.4 (CHAr), 135.5 (CHAr), 137.7 (CAr), 137.9 (C-4), 138.1 (CAr), 158.6 (CAr); HRMS (ESITOF) $m / z:[\mathrm{M}+\mathrm{H}]^{+}$Calcd for $\mathrm{C}_{44} \mathrm{H}_{51} \mathrm{~N}_{2} \mathrm{O}_{2} \mathrm{Si} 667.3714$; Found 667.3711. 
(2aR,6aS,14bS)-4-(Hydroxymethyl)-2,2a,5,6,6a,8,9,14b-octahydro-1H-benz[f]indolo[2,3-a]-

quinolizine (5): First step: Thiophenol $(125 \mu \mathrm{l}, 2.25 \mathrm{mmol})$ and cold TFA $(1.40 \mathrm{~mL})$ were added at 0 ${ }^{\circ} \mathrm{C}$ to compound 17 (30 mg, $0.045 \mathrm{mmol}$ ), and the mixture was stirred for $2 \mathrm{~h}$. The solution was poured into a cold saturated solution of $\mathrm{NaHCO}_{3}$, and the resulting mixture was extracted with $\mathrm{CH}_{2} \mathrm{Cl}_{2}$. The combined organic extracts were dried, filtered, and concentrated. Flash chromatography (4:1 hexaneEtOAc) afforded the deprotected indole derivative (22 mg, 90\%): ${ }^{1} \mathrm{H} \mathrm{NMR}\left(400 \mathrm{MHz}, \mathrm{CDCl}_{3}, \mathrm{COSY}\right.$, $g$-HSQC) $\delta 1.01\left[\mathrm{~s}, 9 \mathrm{H}, \mathrm{C}\left(\mathrm{CH}_{3}\right)_{3}\right], 1.57-1.69(\mathrm{~m}, 2 \mathrm{H}, \mathrm{H}-1), 1.82(\mathrm{~m}, 3 \mathrm{H}, \mathrm{H}-2, \mathrm{H}-5, \mathrm{H}-6), 2.05$ (m, 1H, H-5), 2.28 (m, 2H, H-6, H-8), 2.55 (brs, 1H, H-2a), 2.65 (brs, 1H, H-6a), 2.70 (d, J = 15.2 Hz, 1H, H-9), $2.82(\mathrm{~m}, 1 \mathrm{H}, \mathrm{H}-9), 3.35$ (brd, $J=10.4 \mathrm{~Hz}, 1 \mathrm{H}, \mathrm{H}-14 \mathrm{~b}), 3.47$ (dd, $J=11.2,4.0 \mathrm{~Hz}, 1 \mathrm{H}, \mathrm{H}-8), 4.02(\mathrm{~d}, J=$ 13.2 Hz, 1H, $\mathrm{CH}_{2} \mathrm{OSi}$ ), 4.09 (d, $\left.J=13.2 \mathrm{~Hz}, 1 \mathrm{H}, \mathrm{CH}_{2} \mathrm{OSi}\right), 5.38$ (m, 1H, H-3), 7.07-7.20 (m, 6H, ArH), 7.27-7.32 (m, 4H, ArH), 7.62-7.67 (m, 4H, ArH), 7.69 (brs, 1H, NH); ${ }^{13} \mathrm{C}$ NMR (100.6 MHz, $\left.\mathrm{CDCl}_{3}\right)$ $\delta 19.3\left[\mathrm{C}\left(\mathrm{CH}_{3}\right)_{3}\right], 20.8(\mathrm{C}-5), 22.6(\mathrm{C}-9), 26.8\left[\mathrm{C}\left(\mathrm{CH}_{3}\right)_{3}\right], 26.9$ and $27.1(\mathrm{C}-6, \mathrm{C}-1), 30.1(\mathrm{C}-2), 36.3(\mathrm{C}-$ 2a), 47.4 (C-8), 58.0 (C-6a), 59.8 (C-14b), 67.3 ( $\mathrm{CH}_{2} \mathrm{OSi}$ ), 108.7 (CAr), 110.7 (CHAr), 118.0 (CHAr), 119.3 (CHAr), 121.1 (CHAr), 123.9 (C-3), 127.4 (CHAr), 127.5 (CHAr), 129.4 (CHAr), 129.5 (CHAr), 133.8 (CAr), 134.0 (CAr), 135.4 (CHAr), 135.5 (CHAr), 136.0 (CAr), 137.9 (C-4). Second step: TBAF (55 $\mu \mathrm{l}, 0.055 \mathrm{mmol})$ was added at $0{ }^{\circ} \mathrm{C}$ to a solution of the above deprotected indole derivative ( $15 \mathrm{mg}$, $0.028 \mathrm{mmol})$ in THF $(2.8 \mathrm{~mL})$. The mixture was stirred for $4 \mathrm{~h}$, and then concentrated under reduced pressure. Flash chromatography of the residue afforded pentacyclic alcohol 5 (7 mg, 85\%): mp: 241-242 ${ }^{\circ} \mathrm{C} ;{ }^{1} \mathrm{H}$ NMR (500 MHz, CD $\mathrm{OD}, g$-HSQC) $\delta 1.33(\mathrm{~m}, 1 \mathrm{H}, \mathrm{H}-1), 1.56-1.64(\mathrm{~m}, 1 \mathrm{H}, \mathrm{H}-2), 1.72(\mathrm{td}, J=$ 14.0, 6.0 Hz, 1H, H-6), 1.86-1.96 (m, 2H, H-1, H-5), 2.16-2.22 (m, 2H, H-2, H-5), 2.40 (dm, J = 14.0 Hz, 1H, H-6), 2.46 (m, 1H, H-8), 2.62 (brs, 1H, H-2a), 2.77 (dd, J = 16.0, 4.0 Hz, 1H, H-9), 2.82 (brs, 1H, H-6a), 2.93 (dm, $J=16.0$ Hz, 1H, H-9), 3.56 (brs, 1H, H-14b), 3.68 (m, 1H, H-8), 3.92 (s, 2H, $\left.\mathrm{CH}_{2} \mathrm{OH}\right), 5.39$ (brs, $\left.1 \mathrm{H}, \mathrm{H}-3\right), 6.95(\mathrm{td}, J=8.0,1.5 \mathrm{~Hz}, 1 \mathrm{H}, \mathrm{ArH}), 7.03(\mathrm{td}, J=8.0,1.5 \mathrm{~Hz}, 1 \mathrm{H}, \mathrm{ArH})$, $7.27(\mathrm{~d}, J=7.5 \mathrm{~Hz}, 1 \mathrm{H}, \mathrm{ArH}), 7.37(\mathrm{~d}, J=7.5 \mathrm{~Hz}, 1 \mathrm{H}, \mathrm{ArH})$; when the spectrum was recorded in $\mathrm{CF}_{3} \mathrm{CO}_{2} \mathrm{D}(400 \mathrm{MHz})$, the olefinic proton appeared at $\delta 5.86$ as a broad singlet; ${ }^{13} \mathrm{C} \mathrm{NMR}(125.0 \mathrm{MHz}$, $\left.\mathrm{CD}_{3} \mathrm{OD}\right) \delta 22.1$ (C-5), 22.5 (C-9), 26.9 and 27.3 (C-2, C-6), 30.7 (C-1), 37.3 (C-2a), 48.8 (C-8), 60.8 (C- 
6a), 62.4 (C-14b), 67.7 ( $\left.\mathrm{CH}_{2} \mathrm{OH}\right), 108.2$ (CAr), 111.9 (CHAr), 118.6 (CHAr), 119.8 (CHAr), 122.0 (CHAr), 127.2 (C-3), 128.3 (CAr), 136.0 (CAr), 138.1 (CAr), 139.9 (C-4); m/z (\%) 308 (91, M+), 307 (100, [M-1] $\left.]^{+}\right), 291$ (44), 277 (17), 223 (15), 197 (24), 184 (36), 171 (29), 170 (58), 169 (68), 156 (21), 144 (31); HRMS (ESI-TOF) $m / z:[\mathrm{M}+\mathrm{H}]^{+}$Calcd for $\mathrm{C}_{20} \mathrm{H}_{25} \mathrm{~N}_{2} \mathrm{O}$ 309.1961; Found 309.1954.

\section{Acknowledgement}

Financial support from the MINECO, Spain (Project CTQ2012-35250), and the AGAUR, Generalitat de Catalunya (Grant 2014SGR-155) is gratefully acknowledged. Thanks are also due to the MECD (Spain) for a fellowship to F. A.

\section{Supporting Information Available}

Copies of ${ }^{1} \mathrm{H}$ and ${ }^{13} \mathrm{C}$ NMR spectra of compounds 5, 7, 7', 10-17, and a Table with mass-spectral fragmentations of natural nitraraine and compound 5. This material is available free of charge via the Internet at http://pubs.acs.org.

\section{References}

(1) Ibragimov, A. A.; Yunusov, S. Y. Chem. Nat. Compd. 1985, 21, 502-509.

(2) Ibragimov, A. A.; Yunusov, S. Y. Chem. Nat. Compd. 1985, 21, 510-512.

(3) Tulyaganov, T. S.; Kozimova, N. M. Chem. Nat. Compd. 2005, 41, 578-579.

(4) Tulyaganov, T. S.; Allaberdiev, F. Kh. Chem. Nat. Compd. 2002, 38, 602-604.

(5) Albright, J. D.; Mitsche L. A.; Goldman, L. J. Heterocyclic Compd. 1970, 7, 623-627.

(6) Takano, S.; Samizu, K.; Sugihara, T.; Satoh, S.; Ogasawara, K. Chem. Lett. 1989, 1777-1780.

(7) Sakagami, H.; Ogasawara, K. Heterocycles 2001, 54, 43-47.

(8) (a) Yamaguchi, R.; Hamasaki, T.; Sasaki, T.; Kozima, S.; Takaya, H. Synlett 1991, 719-720.

(b) Yamaguchi, R.; Hamasaki, T.; Sasaki, T.; Ohta, T.; Utimoto, K.; Kozima, S.; Takaya, H. J. Org. Chem. 1993, 58, 1136-1143. 
(9) Salame, R.; Gravel, E.; Poupon, E. Tetrahedron Lett. 2011, 52, 6453-6456.

(10) For a review, see: Gravel, E.; Poupon, E. Nat. Prod. Rep. 2010, 27, 32-56.

(11) Isolation: Duan, J.-A.; Williams, I. D.; Che, C.-T.; Zhou, R.-H.; Zhao, S.-X. Tetrahedron Lett. 1999, 40, 2593-2596.

(12) (a) For a review, see: Amat, M.; Pérez, M.; Bosch, J. Synlett 2011, 143-160. (b) Amat, M.; Gómez-Esqué, A.; Escolano, C.; Santos, M. M. M.; Molins, E.; Bosch, J. J. Org. Chem. 2009, 74, 1205-1211. (c) Amat, M.; Ramos, C.; Pérez, M.; Molins, E.; Florindo, P.; Santos, M. M. M.; Bosch, J. Chem. Commun. 2013, 49, 1954-1956.

(13) For preliminary and related studies, see: Amat, M.; Navío, L.; Llor, N.; Molins, E.; Bosch, J. Org. Lett. 2012, 14, 210-213.

(14) Minor amounts (17\%) of a second cis-diastereoisomer, 7', were also isolated.

(15) For similar $\alpha$-amidoalkylation reactions from $(S)$-tryptophanol-derived lactams lacking the substituent at the aminal carbon under Bischler-Napieralski conditions, see reference $12 \mathrm{~b}$.

(16) This oxidation proved to be difficult, and other methods (Swern, Dess-Martin, IBX) were unsatisfactory. For a review on TPAP as a catalytic oxidant, see: Ley, S. V.; Norman, J.; Griffith, W. P.; Marsden, S. P. Synthesis 1994, 639-666.

(17) Unfortunately, this is the only NMR data reported ${ }^{1}$ for the natural product. 